ENCYCIOPEDDE Encyclopédie berbère

BERBERE

$20 \mid 1998$

20 | Gauda - Girrei

\title{
Géographie linguistique
}

\section{S. Chaker}

\section{OpenEdition}

Journals

Édition électronique

URL : http://journals.openedition.org/encyclopedieberbere/1906

DOI : 10.4000/encyclopedieberbere.1906

ISSN : 2262-7197

\section{Éditeur}

Peeters Publishers

\section{Édition imprimée}

Date de publication : 1 octobre 1998

Pagination : 3059-3061

ISBN : 2-7449-0028-1

ISSN : 1015-7344

\section{Référence électronique}

S. Chaker, "Géographie linguistique », Encyclopédie berbère [En ligne], 20 | 1998, document G30, mis en ligne le 01 juin 2011, consulté le 24 septembre 2020. URL : http://journals.openedition.org/ encyclopedieberbere/1906 ; DOI : https://doi.org/10.4000/encyclopedieberbere.1906

Ce document a été généré automatiquement le 24 septembre 2020

(c) Tous droits réservés 


\section{Géographie linguistique}

\section{S. Chaker}

1 La géographie linguistique est cette branche de la dialectologie qui s'occupe de localiser les unes par rapport aux autres les variations linguistiques, au sein d'une aire linguistique déterminée et de les cartographier. En Europe, cette spécialité a pris son essor à la fin $\mathrm{du} \mathrm{XIX}^{\mathrm{e}}$ siècle et au début $\mathrm{du} \mathrm{Xx}^{\mathrm{e}}$, avec la réalisation des grands atlas systématiques des domaines allemand (Wenker) et français (Gilliéron \& Edmont). Depuis, les travaux de ce type se sont poursuivis et affinés en Europe et les atlas sont régulièrement réactualisés dans tous les grands pays. La technique de base habituellement utilisée consiste à vérifier par enquête de terrain, sur un échantillon de localisations (points d'enquête), un ensemble de formes - en général du vocabulaire et/ ou des phrases typiques (grille d'enquête ou questionnaire), pour reporter ensuite les diverses réalisations sur des cartes. On fait ainsi apparaître la répartition géographique des variations et donc les frontières linguistiques. Pour un paramètre donné, la ligne de délimitation d'une réalisation particulière constitue un isoglosse. Lorsqu'on a un faisceau d'isoglosses, c'est-à-dire lorsque les limites de plusieurs paramètres se superposent ou se rapprochent significativement, on a une frontière entre parlers ou entre dialectes, selon le nombre d'isoglosses et leur importance structurale. Bien sûr, le travail de géographie linguistique peut être plus spécialisé et s'intéresser à des phénomènes linguistiques plus précis, phonétiques ou grammaticaux, qu'il s'agira là aussi de localiser et de cartographier.

2 On comprendra évidemment que les deux difficultés essentielles de la géographie linguistique soient celles du maillage du territoire étudié (choix des points d'enquête) et celles liées à l'élaboration des grilles d'enquêtes (choix des formes linguistiques à soumettre). De ce fait, la géographie linguistique suppose à la fois une excellente connaissance de la géographie et de l'histoire du territoire et une parfaite connaissance de la langue concernée, de ses structures et de ces points de variations potentiels.

Dans le domaine berbère, la géographie linguistique reste, pour l'essentiel, liée au nom d'André Basset, qui a eu une production importante et diversifiée dans ce domaine, avec deux œuvres majeures: Géographie linguistique de la Kabylie (1929) et Atlas linguistiques des parlers berbères (Algérie du nord) (1936/1939). Auxquels s'ajoutent une 
foule d'études plus ponctuelles, sur le domaine saharien et touareg, dont une partie se retrouve dans les Articles de dialectologie berbères (1959). En dehors des travaux de Basset, les recherches dans ce domaine sont rares et plus pointues (cf. Galand 1954).

4 L'œuvre de géographie linguistique de Basset reste donc la référence essentielle mais elle est fortement limitée par le fait qu'il n'a travaillé que le vocabulaire (champ lexical des noms d'animaux domestiques, des parties du corps), du moins pour sa partie publiée car ses archives inédites (Inalco) comportent des approches plus diversifiées. Et les résultats, sur cette base, sont rarement probants en ce sens que le lexique berbère est caractérisé par une très grande dispersion et que la répartition géographie du vocabulaire permet rarement de tracer des frontières stables (les faisceaux d'isoglosses sont exceptionnels). Les variations du lexique ne permettent donc pas d'organiser un territoire en sous-ensembles cohérents. On peut d'ailleurs penser que cette extrême dispersion du vocabulaire berbère a largement déterminé la vision d'André Basset pour qui la langue berbère "s'éparpille directement en plusieurs milliers de parlers locaux " (1952, 1959...).

5 Dépassant cette approche purement lexicaliste, Galand \& Galand-Pernet ont mis en circulation en 1971 un questionnaire linguistique pour le berbère beaucoup plus ample et intégrant les données morpho-syntaxique. S'il a souvent été utilisé par les chercheurs de terrain, il n'en a cependant pas résulté d'étude publiée à caractère systématique.

6 Récemment une recherche menée par un jeune chercheur de l'Université de Béjaïa (Bougie), mais non encore publiée, a sensiblement renouvelé la problématique: Kh. Madoui, après avoir élaboré une grille très complète de critères linguistiques (phonétique, morphologiques, syntaxiques et lexicaux), l'a appliquée en six points de la Petite Kabylie (vallée de la Soummam et côte bougiote). Les résultats sont particulièrement intéressants puisqu'ils mettent en évidence une frontière assez nette entre les parlers de la Kabylie et la Soummam et ceux de la côte méditerranéenne à l'est de Bougie, sur la base sur d'une série d'isoglosses, essentiellement phonétiques et morphologiques. Par ailleurs, il apparait que les indices syntaxiques, en particulier la typologie des syntagmes prédicatifs, ne connaissent pratiquement aucune variation dans cette région - et dans toute la Kabylie d'ailleurs. $\mathrm{H}$ se confirme ainsi que les critères de délimitation des parlers (et des dialectes) sont plutôt à rechercher du côté des données phonéticophonologiques et morpho-syntaxiques que du côté du lexique. Et d'autre part, que l'unité des dialectes reposent avant tout sur le niveau syntaxique (notamment la structure de la phrase simple).

7 Cette recherche devrait donc également contribuer à repenser le problème plus large, ancien et lancinant, de la classification linguistique des dialectes berbères qui, depuis René Basset en passant par Edmond Destaing et A. Laoust et jusqu'aux travaux et propositions récentes de A. Willms (1980), A. Aïkhenvald (1988) ou O. Durand (1991), a fait couler beaucoup d'encre (voir l'excellent synthèse de M. Ameur 1990). Il est probable, comme nous l'envisagions dès 1980 dans notre compte rendu de l'ouvrage de Willms, que c'est sur la base d'une grille d'indices structuraux «lourds» (phonéticophonologiques et morpho-syntaxiques) que l'on pourra classer significativement les différents dialectes berbères. 


\section{BIBLIOGRAPHIE}

AIKHENVALD A., « A Structural and Typological Classification of Berber Languages », Progressive Tradition in African and Oriental Studies, Berlin, 1988, Akademie Verlag.

AMEUR M., « A propos de la classification des dialectes berbères ", Études et documents berbères, 7, 1990, p. 7-27.

BASSET A., Essai de géographie linguistique en Kabylie, Paris, 1929.

BASSET A., Atlas linguistique des parlers berbères. Algérie. Territoires du nord, fasc. I, Equidés, Alger, 1936, fasc. II, Bovins, 1939.

BASSET A., La langue berbère, Londres, IAI, 1952 (1969).

BASSET A. Articles de dialectologie berbère, Paris, 1959, Klincksiek (notamment toute la partie II.

" Géographie linguistique »).

BRETON R., Géographie des langues, Paris, PUF, 1976.

CHAKER S., Compte rendu de : A. Willms, Die dialektale Differenzierung des Berberischen, $m$ Revue de l'Occident Musulman et de la Méditerranée, 34, 1982, p. 154-156.

DAUZAT A., La géographie linguistique, Paris, Flammarion 1922.

DURAND O, «L'enchevêtrement des parlers berbères », Rivista degli Studi Orientali, LXV (3-4), 1991, p. 185-194.

GALAND L., « Un type de frontière linguistique arabe et berbère dans le haouz de Marrakech », Orbis III/1, p. 22-33, 1954.

GALAND L. et Galand-Pernet P., Questionnaire linguistique pour le berbère (vocabulaire et morphosyntaxe), CNRS-LOAB, 16 p., 1971.

GALAND L., « Berbère (La langue) », Encyclopédie de l'Islam, 1.1, Brill, p. 1216-1217, 1960.

GALAND L., " Le berbère ", Les langues dans le monde ancien et moderne, $\left(3^{\mathrm{e}}\right.$ partie : Les langues chamito-sémitiques), Paris, 1988, Éditions du CNRS, p. 207-242.

GILLIERON J. \& EDMONT E., Atlas linguistique de la France, Paris, 1902-1920, 1920.

MADOUI Kh., Contribution à la géographie linguistique de la Petite Kabylie, Mémoire de DEA/Magister (linguistique berbère), Unalco/Université de Béjaïa, 1995. Une synthèse accessible de ce travail se trouve dans :

MADOUI Kh., «Études de géographie linguistique en Petite Kabylie », Études et documents berbères, 14, 1996.

POP S., La dialectologie : aperçu historique et méthodes d'enquêtes linguistiques, I \& II, Louvain. 1951. WILLMS A., Die dialectale Differenzierung des Berberischen, Berlin, 1980, Verlag von Dietrich Reimer, 1980.

INDEX

Mots-clés : Linguistique 